Cahiers de littérature orale

$69 \mid 2011$

Paroles de jeux, paroles de crise

\title{
François LEIMDORFER, Les sociologues et le langage
}

\section{Cécile Leguy}

\section{(2) OpenEdition \\ Journals}

\section{Édition électronique}

URL : https://journals.openedition.org/clo/787

DOI : $10.4000 /$ clo. 787

ISSN : 2266-1816

Éditeur

INALCO

\section{Édition imprimée}

Date de publication : 30 juin 2011

Pagination : $142-145$

ISBN : 978-2-85831-197-2

ISSN : 0396-891X

\section{Référence électronique}

Cécile Leguy, «François LeImdorfer, Les sociologues et le langage », Cahiers de littérature orale [En ligne], 69 | 2011, mis en ligne le 20 février 2013, consulté le 01 juillet 2021. URL : http:// journals.openedition.org/clo/787 ; DOI : https://doi.org/10.4000/clo.787

Ce document a été généré automatiquement le 1 juillet 2021.

\section{(a) (1) ( 8}

Cahiers de littérature orale est mis à disposition selon les termes de la Licence Creative Commons Attribution - Pas d'Utilisation Commerciale 4.0 International. 


\title{
François LEIMDORFER, Les sociologues et le langage
}

\author{
Cécile Leguy
}

\section{RÉFÉRENCE}

François LEIMDORFER, Les sociologues et le langage, Paris, Éditions de la Maison des Sciences de l'Homme, 2010, 290 p. ISBN : 978-2-7351-1333-0

1 François Leimdorfer nous propose ici un ouvrage dense sur les rapports des sociologues au langage, avec un double objectif exposé en introduction : « Ancrer la réflexion sur le langage et le discours dans une perspective sociologique et présenter les points de vue sociologiques de différents auteurs sur le langage, le sens et le discours, qu'ils soient sociologues, anthropologues, historiens, psychologues sociaux, sociolinguistes, analystes de discours» (p. 2), afin de répondre à la question: "En quoi la prise en compte du langage modifie les objets, les méthodes et les résultats de la recherche sociologique?»(idem).

2 La perspective est donc délibérément celle d'un sociologue qui pense et cherche à montrer que sa discipline a tout à gagner à tenir compte des relations entre langage et société. Les titres des différentes parties: "Langage, société et culture ", "Sens et interactions ", « Discours, société, histoire » confirment l'inscription sociologique de l'ouvrage.

3 Si l'on navigue entre les différents courants, le livre ne saurait être conçu comme un manuel : fourmillant de références, jonglant d'une idée à l'autre, d'une théorie à l'autre, il est d'une lecture assez difficile. Il se présente plutôt comme la somme des idées émises sur le langage rassemblées durant une vie de chercheur, une sorte de synthèse, passionnante certes, mais également un peu chaotique. En ce sens, la photo de couverture, de l'auteur, est bien représentative du contenu : on y voit dispersés en tous sens des livres sortis de la bibliothèque du sociolinguiste, du « Que sais-je? » de Pierre Achard à Pierre Bourdieu en passant par La Pensée sauvage de Claude Lévi-Strauss. 
Il faut cependant s'accrocher pour cheminer avec l'auteur à travers les travaux de sociologues ou d'autres auteurs de disciplines diverses qui abordent le langage dans une perspective sociologique. Chaque chapitre est pourvu d'une introduction et d'une synthèse conclusive qui permet de suivre l'auteur dans sa progression, malgré la densité du propos. F. Leimdorfer prend le parti de rendre compte de la complexité des propositions et de leur dimension réflexive et s'attache dans un premier temps à « dégager les lieux, les thèmes et les concepts qui font le lien entre l'espace du langage et celui de la société et de dessiner les différents "paradigmes" sous-jacents organisateurs de la réflexion sociologique » (p.13).

5 Après un premier chapitre consacré à des concepts généraux, envisageant le langage sous son double aspect d'action et de signification, l'objet des chapitres formant la première partie est la question des rapports entre langage, société et culture, dans une perspective différenciée d'abord (premier paradigme, chapitres 2 et 3), langage et société ayant leur propre logique, mais entraînant des effets l'un sur l'autre. Le chapitre 2 envisage les faits de langage comme fondés sur les rapports sociaux, dans une perspective durkheimienne (mise en parallèle avec la conception marxiste) qui est celle de linguistes du début $\mathrm{du} \mathrm{xx}^{\mathrm{e}}$ siècle comme Antoine Meillet par exemple, ou encore beaucoup plus tard celle de Bourdieu mettant en valeur le rôle des rapports sociaux de domination dans les usages langagiers. Dans ce chapitre sont traités les concepts d'idéologie et de représentation; le langage y est présenté comme étant l'expression d'« autre chose » (p. 56). Dans le chapitre 3, F. Leimdorfer explore cette conception du reflet du point de vue des anthropologues qui lui semblent avoir une même vision globalisante, mais inverse, en considérant à la suite de Franz Boas, Edward Sapir et Benjamin Lee Whorf, que «le langage imprime sa marque sur la culture» (p.59). Ce chapitre, qui rassemble sous une même problématique l'hypothèse Sapir-Whorf, le structuralisme de Lévi-Strauss et Calame-Griaule - dont les travaux sont présentés de manière un peu confuse - peut sembler bien hétéroclite. Le quatrième chapitre envisage un deuxième paradigme, où langage et société ne sont plus conçus comme miroir l'un de l'autre, mais comme un ensemble unifié, abordé à partir des situations sociales de communication. Cette perspective est représentée d'une part par Bronislaw Malinowski et le point de vue pragmatique qu'il développe, considérant le langage comme une activité, d'autre part par l'ethnographie de la communication telle qu'elle s'est définie autour de Dell Hymes et de John J. Gumperz dans les années 1960. Ce sont les liens fondamentaux entre faits de langue et faits sociaux qui sont ici mis en valeur. Dans le chapitre suivant, c'est plus précisément la corrélation entre catégories linguistiques et catégories sociales qui est envisagée, avec la linguistique variationniste de William Labov et les travaux sur les différences entre codes élaboré et restreint de Basil Bernstein.

6 La deuxième partie, intitulée "Sens et interactions ", comprend trois chapitres. La perspective de départ est celle de la sociologie du sens, telle que l'ont engagée les sociologues allemands Georg Simmel et Max Weber et, à leur suite, l'École de Chicago et l'interactionnisme symbolique. Leur point de vue est plus local, centré sur la situation, mais ils ne développent pas à proprement parler d'analyse du sens à partir de productions langagières effectives. Cela sera fait après la Seconde Guerre mondiale, entre autres par Erving Goffman et les ethnométhodologues - auxquels est consacré le chapitre 7 - qui vont plus précisément s'intéresser aux situations ordinaires. Pour clore cette deuxième partie, le chapitre 8 aborde les notions de situation et de conversation. 
F. Leimdorfer voit se dégager, dans cette manière d'aborder la réalité sociale à partir de situations locales d'interaction, un troisième paradigme des rapports entre faits de langage et faits sociaux où tout se joue en situation, le sujet n'étant pas isolable des autres, mais participant avec eux à la « coconstruction des activités et des discours » (p. 159).

7 La troisième partie envisage enfin les relations entre discours, société et histoire. Il s'agit pour F. Leimdorfer d'aborder l'énonciation « comme articulation entre l'espace $\mathrm{du}$ sociohistorique et l'espace du linguistique, et les catégories de discours et les espaces discursifs comme institutions sociales et historiques structurantes » (p. 14). Le discours, dont l'auteur donne une définition précise selon trois perspectives différentes (p. 167-171), est l'espace où se jouent les rapports entre le social et le langagier. Le chapitre 9 traite des formes du discours, du dialogisme de Mikhail Bakhtine aux genres de discours, tandis que le chapitre 10 porte sur la dimension temporelle du discours quand il se fait récit des événements, Histoire. Le rôle de l'écrit, tel qu'il a été présenté par Jack Goody, est alors envisagé dans la mesure où l'écriture est conçue comme condition de l'Histoire. L'écrit mène au discours, tel qu'il peut être abordé par Michel Foucault, comme condition de possibilité d'un savoir, propre à une époque (p. 196), et c'est cette définition foucaldienne qui est retenue par F. Leimdorfer pour traiter des formations discursives et de l'ordre du discours. Cependant, pour analyser les discours, c'est la linguistique qui fournit concepts et méthodes, comme il le montre dans le chapitre 11. La sociologie gagne à recourir à l'analyse de discours (ici, il s'agit surtout de celles de Michel Pêcheux et de Pierre Achard), non seulement parce que la question du sens l'intéresse, mais aussi parce qu'il est important de « situer la place du langage et du discours dans les rapports sociaux [de] dessiner les effets du langage sur ceux-ci » (p. 227). Aussi, F. Leimdorfer affirme-t-il que le langage n'est pas un simple domaine à prendre en compte en sociologie, mais qu'il en est « l'instrument principal » (p. 250). Dans le douzième et dernier chapitre, l'auteur propose enfin de mettre en valeur les lignes théoriques de ce qu'il présente comme un quatrième paradigme du rapport entre langagier et sociologique, à partir du discours entendu comme activité sociale, lieu de l'interdépendance du langagier et du social.

8 «La prise en compte du langage en sociologie doit être rigoureuse et permettre de nouvelles avancées, notamment en considérant le discours comme une activité. Elle ne doit pas se limiter, comme il est si souvent fait, à des commentaires sur des textes et des entretiens ou à considérer ce qui est dit comme une information, dans une transparence du langage au réel» (p. 252). On ne peut que suivre ces propos militants de l'auteur, faisant avec force la preuve que les sociologues ne sauraient ignorer les faits de langage. 